\title{
ON TRANSLATIONS OF FUNCTIONS AND SETS ${ }^{1}$
}

\section{RALPH PALMER AGNEW}

1. Introduction. It is the object of this note to prove the following theorem and two lemmas (see \$3) on translations of sets which are used in the proof of the theorem.

THEOREM 1. In order that a sequence $x_{n}(t)$ of complex-valued functions measurable over $-\infty<t<\infty$ may be such that, for each real sequence $\lambda_{n}$,

$$
\lim _{n \rightarrow \infty} x_{n}\left(t-\lambda_{n}\right)=0
$$

for almost all $t$, it is necessary and sufficient that for each $\delta>0$

$$
\sum_{n=1}^{\infty} \text { l.u.b. }\left|E_{t}\left\{h \leqq t \leqq h+1 ;\left|x_{n}(t)\right| \geqq \delta\right\}\right|<\infty .
$$

Necessity for Theorem 1 is established by proving the following more incisive theorem.

THEOREM 2. If a sequence $x_{n}(t)$ of complex-valued functions measurable over $-\infty<t<\infty$ is such that, for each real sequence $\lambda_{n}$,

$$
\lim _{n \rightarrow \infty} x_{n}\left(t-\lambda_{n}\right)=0
$$

for each $t$ in some set $D$ of positive measure (where the set $D$ may depend upon the sequence $\lambda_{n}$ ), then (2) holds.

Measure is that of Lebesgue, and a property such as (1) holds for almost all $t$ if it holds for all $t$ in the infinite interval $-\infty<t<\infty$ with the possible exception of a null set (set of measure 0 ). The set

$$
A \equiv A(h, t, n, \delta)=E_{t}\left\{h \leqq t \leqq h+1 ;\left|x_{n}(t)\right| \geqq \delta\right\}
$$

is the set of all points $t$ such that $h \leqq t \leqq h+1$ and $\left|x_{n}(t)\right| \geqq \delta$; and $|A|$ denotes the measure of $A$. The condition (2) implies that when $n$ is large the function $\left|x_{n}(t)\right|$ is less than $\delta$ for "most" values of $t$ in each unit interval; but (2) implies no restriction whatever on $x_{n}(t)$ when $t$ lies in the "exceptional" set.

The hypothesis that (1) holds for almost all $t$ for each real bounded sequence $\lambda_{n}$ does not imply (2). For example if, for each $n=1,2,3, \cdots, x_{n}(t)$ is a constant $c_{n}$ over the interval $2^{n}<t<2^{n}+1$ and is 0 otherwise, and $\lambda_{n}$ is a bounded sequence, then (1) holds for

\footnotetext{
${ }^{1}$ Presented to the Society, September 8, 1939.
} 
each $t$; but (2) fails in case $c_{n}$ fails to converge to 0 as $n$ becomes infinite.

2. Proof of sufficiency for Theorem 1. Let $x_{n}(t)$ be a sequence of measurable functions for which (2) holds, and let $\lambda_{n}$ be a sequence of real numbers. It follows from (2) that, for each $\delta>0$,

$$
\sum_{n=1}^{\infty} \underset{-\infty<h<\infty}{\text { l.u.b. }}\left|E_{t}\left\{h \leqq t \leqq h+1 ;\left|x_{n}\left(t-\lambda_{n}\right)\right| \geqq \delta\right\}\right|<\infty .
$$

Let $J$ denote an arbitrary finite interval. Since $J$ can be covered by a finite set of unit intervals $h \leqq t \leqq h+1$, it follows from (3) that for each $\delta>0$

$$
\sum_{n=1}^{\infty}\left|E_{t}\left\{t \varepsilon J ;\left|x_{n}\left(t-\lambda_{n}\right)\right| \geqq \delta\right\}\right|<\infty \text {. }
$$

Setting

$$
A_{n, p}=E_{t}\left\{t \varepsilon J ;\left|x_{n}\left(t-\lambda_{n}\right)\right| \geqq p^{-1}\right\}, \quad n, p=1,2,3, \cdots,
$$

we see that (4) implies existence of indices $n_{1}<n_{2}<n_{3}<\cdots$ such that

$$
\sum_{n=n_{p}}^{\infty}\left|A_{n, p}\right|<2^{-p-1}, \quad p=1,2, \cdots .
$$

Setting

$$
A_{r}=\sum_{p=r}^{\infty} \sum_{n=n_{p}}^{\infty} A_{n, p}, \quad r=1,2, \cdots,
$$

we find

$$
\left|A_{r}\right| \leqq \sum_{p=r}^{\infty} \sum_{n=n_{p}}^{\infty}\left|A_{n, p}\right|<\sum_{p=r}^{\infty} 2^{-p-1}=2^{-r}, \quad r=1,2, \cdots .
$$

Let

$$
J_{r}=J-A_{r}, \quad r=1,2, \cdots
$$

If $t \varepsilon J_{r}$ then, when $p>r$,

$$
\left|x_{n}\left(t-\lambda_{n}\right)\right|<p^{-1}, \quad n \geqq n_{p},
$$

so that $x_{n}\left(t-\lambda_{n}\right)$ converges to 0 over $J_{r}$. Hence $x_{n}\left(t-\lambda_{n}\right)$ converges to 0 over $J_{1}+J_{2}+\cdots$. But $J_{r}$ is a subset of $J$ having measure greater than $|J|-2^{-r}$; hence $J_{1}+J_{2}+\cdots$ is a subset of $J$ having measure $|J|$. Therefore $x_{n}\left(t-\lambda_{n}\right)$ converges to 0 for almost all $t$ in $J$. 
Since $J$ is an arbitrary finite interval, $x_{n}\left(t-\lambda_{n}\right)$ must converge to 0 for almost all $t$ in $-\infty<t<\infty$ and sufficiency for Theorem 1 is proved.

3. Lemmas on translations of sets. In this section we prove two lemmas. The first states that if $C$ and $B$ are measurable subsets of unit intervals, then it is possible to translate $B$ in such a way that the intersection of $C$ and the translation of $B$ will have measure at least $\frac{1}{2}|C||B|$. The first lemma is used in proof of the second which specifies conditions under which a given sequence of sets can be translated so as to cover each point of the interval $-\infty<t<\infty$, with the exception of a null set, an infinite number of times. The close connection established in $\$ 4$ between Lemma 2 and Theorem 2 shows that the combined proofs of Lemmas 1 and 2 furnish essentially a proof of Theorem 2.

If $E$ is a set of points $t$ in the interval $-\infty<t<\infty$ and $\lambda$ is a real number, let $E(\lambda)$ denote the set of points $t$ such that $t-\lambda \varepsilon E$; thus $E(\lambda)$ is the set obtained by translating the set $E$ to the right $\lambda$ units. Let $U$ denote the unit interval $0 \leqq t \leqq 1$.

Lemma 1. If $C$ and $B$ are measurable subsets of $U$, then

$$
\max _{-1 \leqq \lambda \leqq 1}|C B(\lambda)| \geqq \frac{1}{2}|C||B| .
$$

Let $\phi(t)$ be the characteristic function of $C$, that is, $\phi(t)=1$ when $t_{\varepsilon} C$ and $\phi(t)=0$ otherwise; and let $\psi(t)$ be the characteristic function of $B$. Then $\psi(t-\lambda)$ is the characteristic function of $B(\lambda)$, and $\phi(t) \psi(t-\lambda)$ is the characteristic function of the intersection $C B(\lambda)$ of $C$ and $B(\lambda)$. Hence on denoting the measure of $C B(\lambda)$ by $\mu(\lambda)$ we have

$$
\mu(\lambda)=\int_{-\infty}^{\infty} \phi(t) \psi(t-\lambda) d t .
$$

The function $\mu(\lambda)$ is continuous since

$$
\begin{aligned}
|\mu(\lambda+h)-\mu(\lambda)| & \leqq \int_{-\infty}^{\infty} \phi(t)|\psi(t-\lambda-h)-\psi(t-\lambda)| d t \\
& \leqq \int_{-\infty}^{\infty}|\psi(t-\lambda-h)-\psi(t-\lambda)| d t \\
& =\int_{-\infty}^{\infty}|\psi(t-h)-\psi(t)| d t
\end{aligned}
$$

and the last integral converges to 0 with $h$. Hence $\mu(\lambda)$ has a maxi- 
mum over the interval $-1 \leqq \lambda \leqq 1$. Since $\mu(\lambda)=0$ when $|\lambda|>1$, the computation

$$
\begin{aligned}
\int_{-1}^{1} \mu(\lambda) d \lambda & =\int_{-\infty}^{\infty} d \lambda \int_{-\infty}^{\infty} \phi(t) \psi(t-\lambda) d t \\
& =\int_{-\infty}^{\infty} \phi(t) d t \int_{-\infty}^{\infty} \psi(t-\lambda) d \lambda=|C||B|
\end{aligned}
$$

is easily justified. This equality and the inequality

$$
\mu(\lambda) \leqq \max _{-1 \leqq \lambda \leqq 1} \mu(\lambda), \quad-1 \leqq \lambda \leqq 1,
$$

imply that $\max |C B(\lambda)|=\max \mu(\lambda) \geqq \frac{1}{2}|C||B|$ and Lemma 1 is established.

The fact that use of inequalities such as (13) often leads to crude results may make one suspicious that Lemma 1 holds when the factor $\frac{1}{2}$ in (11) is replaced by a greater factor. To settle this question, let $0<\epsilon<\frac{1}{3}$, let $C=E_{t}\{\epsilon \leqq t \leqq 1-\epsilon\}$, and let $B=E_{t}\{0 \leqq t \leqq \epsilon\}$ $+E_{t}\{1-\epsilon \leqq t \leqq 1\}$. Then $|C|=1-2 \epsilon,|B|=2 \epsilon$, and it is easy to verify that

$$
\max _{-1 \leqq \lambda \leqq 1}|C B(\lambda)|=\epsilon=[1 /(2-4 \epsilon)]|C||B|>0 .
$$

This shows that $\frac{1}{2}$ is the greatest factor permissible in (11).

LEMma 2. If $A_{1}, A_{2}, \cdots$ is a sequence of measurable sets and a sequence $U_{1}, U_{2}, \cdots$ of unit intervals exists such that

$$
\sum_{n=1}^{\infty}\left|U_{n} A_{n}\right|=\infty
$$

then there exists a sequence $\lambda_{1}, \lambda_{2}, \cdots$ such that each $t$ in the interval $-\infty<t<\infty$, except those in some null set, lies in an infinite number of the sets $A_{n}\left(\lambda_{n}\right)$.

Let $B_{n}=U_{n} A_{n}$ so that each $B_{n}$ lies in some unit interval and $\sum\left|B_{n}\right|=\infty$. Let $n$ be fixed. Choose $\lambda_{n}$ such that $B_{n}\left(\lambda_{n}\right) \subset U$, where $U$ is as before the unit interval $0 \leqq t \leqq 1$, and let $C_{n}=U-B_{n}\left(\lambda_{n}\right)$. Since $\lambda_{n+1}^{\prime}$ exists such that $B_{n+1}\left(\lambda_{n+1}^{\prime}\right) \subset U$, Lemma 1 guarantees existence of $\lambda_{n+1}$ such that

$$
\left|C_{n} B_{n+1}\left(\lambda_{n+1}\right)\right| \geqq \frac{1}{2}\left|C_{n}\right|\left|B_{n+1}\right| \text {. }
$$

Let $C_{n+1}=U-\left[B_{n}\left(\lambda_{n}\right)+U B_{n+1}\left(\lambda_{n+1}\right)\right]$. Again from Lemma $1, \lambda_{n+2}$ exists such that (16) holds when $n$ is replaced by $n+1$. In this manner, 
we obtain a sequence $\lambda_{n}, \lambda_{n+1}, \cdots$ of real numbers and a sequence

$$
C_{n+p}=U-\left[U B_{n}\left(\lambda_{n}\right)+U B_{n+1}\left(\lambda_{n+1}\right)+\cdots+U B_{n+p}\left(\lambda_{n+p}\right)\right]
$$

of sets such that, for each $p=0,1,2, \cdots$,

$$
\sum_{k=n}^{n+p}\left|C_{k} B_{k+1}\left(\lambda_{k+1}\right)\right| \geqq \frac{1}{2} \sum_{k=n}^{n+p}\left|C_{k}\right|\left|B_{k+1}\right| \text {. }
$$

Since the sets $C_{k} B_{k+1}\left(\lambda_{k+1}\right)(k=n, n+1, \cdots, n+p)$ are subsets of $U$ and no two have a point in common, the left member of (18) is less than or equal to unity for each $p=0,1,2, \cdots$. From this it follows that $\left|C_{n+p}\right| \rightarrow 0$ as $p \rightarrow \infty$; for $\left|C_{n+p}\right|$ is monotone decreasing as $p \rightarrow \infty$ and if $\left|C_{n+p}\right|$ is bounded from 0 , then the fact that $\sum\left|B_{n}\right|=\infty$ would imply that the right member of (18) diverges to $+\infty$ as $p \rightarrow \infty$. The conclusion that $\left|C_{n+p}\right| \rightarrow 0$ as $p \rightarrow \infty$ implies by (17) that

$$
\lim _{p \rightarrow \infty}\left|U B_{n}\left(\lambda_{n}\right)+U B_{n+1}\left(\lambda_{n+1}\right)+\cdots+U B_{n+p}\left(\lambda_{n+p}\right)\right|=1 .
$$

Hence there exists a sequence $0=n_{1}<n_{2}<\cdots$ of indices such that the set

$$
D_{k} \equiv U B_{n_{k}+1}\left(\lambda_{n_{k}+1}\right)+\cdots+U B_{n_{k+1}}\left(\lambda_{n_{k+1}}\right)
$$

has measure $\left|D_{k}\right|>1-2^{-k-1}$ for each $k=1,2, \cdots$. Put $P_{k}=D_{k} D_{k+1} \cdots$ and $P=P_{1}+P_{2}+\cdots$. The fact that $D_{k} \subset U$ and $\left|D_{k}\right|>1-2^{-k-1}$ for each $k=1,2, \cdots$ implies that $P_{k} \subset U$ and $\left|P_{k}\right| \geqq 1-2^{-k}$, and consequently $P \subset U$ and $|P|=1$. If $t \varepsilon P$, then $t \varepsilon P_{k}$ for some $k$ so that $t \varepsilon D_{k}$ for all sufficiently great $k$ and $t \varepsilon B_{n}\left(\lambda_{n}\right)$ for an infinite set of $n$, and hence also $t \varepsilon A_{n}\left(\lambda_{n}\right)$ for an infinite set of $n$.

If the sequence of sets $A_{n}$ is arranged in a double sequence $A_{p, q}$ $(p=0, \pm 1, \cdots ; q=1,2, \cdots)$ in such a way that

$$
\sum_{q=1}^{\infty}\left|A_{p, q}\right|=\infty, \quad p=0, \pm 1, \pm 2, \cdots,
$$

it results from what we have already proved that for each fixed $p$ there is a sequence $\lambda_{p, 1}, \lambda_{p, 2}, \cdots$ such that each point of a subset of $I_{p} \equiv E_{t}\{p \leqq t \leqq p+1\}$ of measure unity is contained in an infinite number of the sets $A_{p, 1}\left(\lambda_{p, 1}\right), A_{p, 2}\left(\lambda_{p, 2}\right), \cdots$. Then each point of $-\infty<t<\infty$ with the exception of a null set lies in an infinite number of sets of the double sequence $A_{p, q}\left(\lambda_{p, q}\right)$ which can be arranged in the simple sequence $A_{n}\left(\lambda_{n}\right)$, and proof of Lemma 2 is complete.

The hypothesis of Lemma 2 is equivalent to the following: $A_{n}$ is a sequence of measurable sets such that 


$$
\sum_{n=1}^{\infty} \underset{-\infty<h<\infty}{\text { l.u.b. }}\left|E_{t}\left\{h \leqq t \leqq h+1 ; t \varepsilon A_{n}\right\}\right|=\infty .
$$

That the hypothesis (22) cannot be relaxed is a consequence of the following result which we give without proof. If $A_{1}, A_{2}, \ldots$ is a sequence of measurable sets, and a real sequence $\lambda_{1}, \lambda_{2}, \cdots$ and a set $C$ of positive measure exist such that each point of $C$ lies in an infinite number of the sets $A_{n}\left(\lambda_{n}\right)$, then (22) holds.

That the conclusion of Lemma 2 must provide for an exceptional null set becomes clear when one observes that if the sets $A_{n}$ are each nondense then, however $\lambda_{1}, \lambda_{2}, \cdots$ are determined, the set $\sum A_{n}\left(\lambda_{n}\right)$ must be of the first category and hence there must be a set of the second category whose points are in none of the sets $A_{n}\left(\lambda_{n}\right)$.

4. Proof of Theorem 2. To prove Theorem 2, let $x_{n}(t)$ be a sequence of measurable functions for which (2) fails for some $\delta>0$. Then $\delta>0$ and a sequence $h_{1}, h_{2}, \cdots$ exist such that

$$
\sum_{n=1}^{\infty} E_{t}\left\{h_{n} \leqq t \leqq h_{n}+1 ;\left|x_{n}(t)\right| \geqq \delta\right\}=\infty .
$$

Let $A_{n}=E_{t}\left\{\left|x_{n}(t)\right| \geqq \delta\right\}$. Then by Lemma 2 there exist a sequence $\lambda_{1}, \lambda_{2}, \cdots$ and a set $C$ whose complement is a null set such that each $t$ in $C$ lies in an infinite number of the sets $A_{n}\left(\lambda_{n}\right)$. Hence if $t \varepsilon C$, then $t-\lambda_{n} \varepsilon A_{n}$ for an infinite set of $n$ so that $\left|x_{n}\left(t-\lambda_{n}\right)\right| \geqq \delta$ for an infinite set of $n$. This contradicts the hypothesis of Theorem 2 and completes the proof.

\section{Cornell University}

